Midplane-symmetry breaking in the flow between two counter-rotating disks

Hewitt, R.E. and Hazel, A.L.

2007

MIMS EPrint: 2012.22

Manchester Institute for Mathematical Sciences

School of Mathematics

The University of Manchester

\footnotetext{
Reports available from: http://eprints.maths.manchester.ac.uk/

And by contacting: The MIMS Secretary

School of Mathematics

The University of Manchester

Manchester, M13 9PL, UK
} 


\title{
Midplane-symmetry breaking in the flow between two counter-rotating disks
}

\author{
Richard E. Hewitt • Andrew L. Hazel
}

Received: 23 May 2006 / Accepted: 15 September 2006 / Published online: 10 November 2006

(C) Springer Science+Business Media B.V. 2006

\begin{abstract}
This paper considers the axisymmetric steady flow driven by exact counter rotation of two co-axial disks of finite radius. At the edges of the rotating disks one of three conditions is (typically) imposed: (i) zero velocity, corresponding to a stationary, impermeable, cylindrical shroud (ii) zero normal velocity and zero tangential fluid traction, corresponding to a (confined) free surface and (iii) an edge constraint that is consistent with a similarity solution of von Kármán form. The similarity solution is valid in an infinite geometry and possesses a pitchfork bifurcation that breaks the midplane symmetry at a critical Reynolds number. In this paper, similar bifurcations of the global (finite-domain) flow are sought and comparisons are made between the resulting bifurcation structure and that found for the similarity solution. The aim is to assess the validity of the nonlinear similarity solutions in finite domains and to explore the sensitivity of the solution structure to edge conditions that are implicitly neglected when assuming a self-similar flow. It is found that, whilst the symmetric similarity solution can be quantitatively useful for a range of boundary conditions, the bifurcated structure of the finite-domain flow is rather different for each boundary condition and bears little resemblance to the self-similar flow.
\end{abstract}

Keywords Rotating disk $\cdot$ Bifurcation $\cdot$ Similarity solution

\section{Introduction}

The axisymmetric flow between rotating disks has been a topic of significant interest for several decades. As first noted by Batchelor [1], the flow supports similarity solutions of von Kármán type, in which the radial and azimuthal flow velocities increase linearly with distance from the axis of rotational symmetry, whilst the transverse velocity is independent of this distance. Such flows are explored in great detail in the review article of Zandbergen and Dijkstra [2].

Von Kármán flow is one of a class of exact stagnation-type solutions to the Navier-Stokes equations. The term "exact" in this context signifies that the assumed form of solution reduces the steady NavierStokes equations to a much simpler ordinary-differential, boundary-value problem without requiring any approximation; that is, no terms are neglected. There are many analogous flows with the same stagnation

R. E. Hewitt $(\bowtie) \cdot$ A. L. Hazel

School of Mathematics, The University of Manchester, Oxford Road, Manchester, M13 9PL, UK

e-mail: richard.e.hewitt@manchester.ac.uk 
form, for example the axisymmetric motion between two uniformly porous disks, or two "stretching" disks in which the velocity is directed radially out/inwards in proportion to the distance from the axis of symmetry. Equivalent two-dimensional solutions in Cartesian geometries include the flow in a porous channel driven by uniform wall transpiration [3], or the flow in a stretching channel [4]; and these flows can easily be extended to three dimensions $[5,6]$. Furthermore, the same form of solution can be applied to cylindrical pipe flow, again driven by uniform transpiration [7] or with axial stretching [4]. In all these cases the velocity components are scaled at most linearly by a spatial coordinate. Hence, these solutions can be made unsteady without breaking the spatial self-similarity.

The flows described above have been applied to far too many problems to list here; see the recent monograph by Riley and Drazin [8] for a review. Initially, the flow states themselves were the topic of interest, owing to the exact nature of the solutions in the broader framework of the Navier-Stokes equations. In many cases, however, the similarity solutions are introduced on the basis of a large aspect ratio being present in the problem under consideration. An implicit assumption is that in a domain of large aspect ratio, the far-field boundary conditions only have local influence and can be neglected, allowing the self-similar solution to be used to describe a finite region of the near-axis flow. In other words, there is an implicit assumption of Saint-Venant's principle. For these similarity solutions, we may also note that bifurcations and non-uniqueness are commonplace. It is typically assumed that the nonlinear behaviour represented in the self-similar equations will be replicated in a global solution of the Navier-Stokes equations in some finite domain.

For the flow between two co-axial rotating disks, Brady and Durlofsky [9] examined the relationship between the von Kármán solutions and solutions of the parabolized Navier-Stokes equations in large but finite axial domains. Their conclusions were that end effects can be important even in arbitrarily large (but finite) domains. Using a Reynolds number, Re, defined by choosing the distance between the two disks as the natural length scale, they noted that what quantitative agreement there was (between the similarity and "finite-disk" solutions) rarely extend beyond the first $20 \%$ of the flow domain for $\mathrm{Re} \geq 200$. The authors claimed that qualitative agreement was available over a broader range and that the similarity solution was, therefore, of use as an approximation even at moderate to high Reynolds numbers. Most significantly, in the context of the present work, they reported that, in the case of exact counter-rotation of the disks, "we find the solution to be unique at all values of the Reynolds number considered", a result in contrast to the behaviour of the similarity solution, which possess a (midplane) symmetry breaking bifurcation.

In the present work, we further develop these ideas by solving the axisymmetric Navier-Stokes equations numerically over a domain of fixed aspect ratio. Previous numerical and experimental studies of such rotating-disk flows have revealed rich and varied dynamics, see e.g. $[10,11]$ and the references therein. Even so, the three-dimensional parameter space spanned by the Reynolds number, the aspect ratio of the domain and relative rotation rates of the two disks has not been comprehensively explored. We chose to concentrate exclusively on the case of exact counter-rotation, in which the two disks rotate at the same frequency but in opposite directions. This configuration admits solutions that possess a reflectional symmetry with respect to the midplane, and, as mentioned above, the similarity solution undergoes a supercritical pitchfork bifurcation in this configuration. Our (broad) aims for this paper are to address some simple questions: (i) does the finite-domain system undergo a pitchfork bifurcation at large aspect ratios? (ii) if so, are the similarity solution predictions for the critical Reynolds number and eigenmode useful in finite (but large) domains? (iii) and generally, to what extent are the similarity solutions accurate descriptions of the near-axis flow.

Partial answers to some of these questions are already available in the literature, but the picture is still rather incomplete because each study has tended to concentrate on one particular set of boundary conditions and relative rotation ratio. One such condition is a closed-end, appropriate for a configuration in which the disks are enclosed by a fixed, impermeable shroud. For this condition, Nore et al. [11] conducted a numerical investigation of the stability of exact counter-rotating disk flow at small aspect ratios; until this point, counter rotation had received relatively little attention. They found a midplane-symmetry 
breaking pitchfork bifurcation that did not coincide with that of the similarity solution. For the aspect ratios considered, however, stability of the primary symmetric state was first lost through bifurcations to non-axisymmetric flows. For alternative, open-end boundary conditions, defined below, Witkowski et al. [12] examined the nature of the first axisymmetric bifurcation in domains of large aspect ratio. Once again, the location of the bifurcation did not coincide with that of the similarity solution. Moreover, at large aspect ratios, they found that the symmetric, axisymmetric solution first becomes unstable through a Hopf bifurcation at low values of the Reynolds number.

In order to examine end effects in some detail, we apply three different types of boundary condition at the disk edges. In the first (closed-end) case, we apply no-slip boundary conditions to model the case when the disks are surrounded by a stationary, impermeable cylindrical shroud. In the second (open-end) case, a traction constraint is imposed that corresponds to a stationary "free surface". In the final case, conditions consistent with the similarity solution are imposed at the edge of the flow domain. The use of homotopy continuation techniques allows us to explore the connection between the behaviour of the system under different boundary conditions and the relationship between the similarity solution and solutions in the finite domain.

\section{Formulation}

We consider a viscous, incompressible, fluid of constant density $\rho$ and dynamic viscosity $\mu$ confined by two identical disks, each of radius $L$, separated by a distance $h$. The disks are aligned co-axially and counterrotate with constant angular frequencies $\pm \omega$. The flow is described using a cylindrical polar coordinate system $\left(r^{*}, \theta, z^{*}\right)$, where $r^{*}=0$ is the axis of rotation and $z^{*}=0$ is the midplane of the flow domain. Here, an asterisk is used to distinguish dimensional quantities from their dimensionless equivalents.

The problem is non-dimensionalised by choosing $h$ and $\omega h$ as the natural length and velocity scales, respectively, giving a Reynolds number, $\mathrm{Re}=\rho \omega h^{2} / \mu$. The dimensionless coordinates are $r=r^{*} / h \in[0, \Gamma]$ and $z=z^{*} / h \in[-1 / 2,1 / 2]$, where $\Gamma=L / h$ is the aspect ratio of the domain. The dimensionless velocity field is $\boldsymbol{u}=\boldsymbol{u}^{*} /(\omega h)$, with components $(u, v, w)$ in the directions of increasing $(r, \theta, z)$, respectively; and the fluid pressure, $p^{*}$, is non-dimensionalised on the viscous scale, $p=p^{*} /(\mu \omega)$.

If the aspect ratio, $\Gamma$, is large, then it is convenient to introduce a re-scaled radial coordinate $\hat{r}=r / \Gamma \in$ $[0,1]$ and corresponding radial and azimuthal velocity components $\hat{u}=u / \Gamma, \hat{v}=v / \Gamma$. For notational convenience, we relabel the unchanged vertical coordinate, vertical velocity component and fluid pressure, $\hat{z}=z, \hat{w}=w$ and $\hat{p}=p$.

The governing equations in this rescaled problem are the steady, axisymmetric, Navier-Stokes equations in cylindrical polar coordinates:

$$
\begin{aligned}
& \operatorname{Re}\left[\hat{u} \frac{\partial \hat{u}}{\partial \hat{r}}-\frac{\hat{v}^{2}}{\hat{r}}+\hat{w} \frac{\partial \hat{u}}{\partial \hat{z}}\right]=-\frac{1}{\Gamma^{2}} \frac{\partial \hat{p}}{\partial \hat{r}}+\mathcal{D}^{2} \hat{u}, \\
& \operatorname{Re}\left[\hat{u} \frac{\partial \hat{v}}{\partial \hat{r}}+\frac{\hat{u} \hat{v}}{\hat{r}}+\hat{w} \frac{\partial \hat{v}}{\partial \hat{z}}\right]=\mathcal{D}^{2} \hat{v}, \\
& \operatorname{Re}\left[\hat{u} \frac{\partial \hat{w}}{\partial \hat{r}}+\hat{w} \frac{\partial \hat{w}}{\partial \hat{z}}\right]=-\frac{\partial \hat{p}}{\partial \hat{z}}+\nabla^{2} \hat{w},
\end{aligned}
$$

where

$\nabla^{2} f \equiv \frac{1}{\Gamma^{2}}\left(\frac{\partial^{2} f}{\partial \hat{r}^{2}}+\frac{1}{\hat{r}} \frac{\partial f}{\partial \hat{r}}\right)+\frac{\partial^{2} f}{\partial \hat{z}^{2}} \quad$ and $\quad \mathcal{D}^{2} f \equiv \nabla^{2} f-\frac{1}{\Gamma^{2}} \frac{f}{\hat{r}^{2}}$

and the continuity equation

$\frac{1}{\hat{r}} \frac{\partial}{\partial \hat{r}}(\hat{r} \hat{u})+\frac{\partial \hat{w}}{\partial \hat{z}}=0$. 
The no-slip and impermeability boundary conditions on the counter-rotating disks are:

$\hat{u}\left(\hat{r}, \hat{z}= \pm \frac{1}{2}\right)=\hat{w}\left(\hat{r}, \hat{z}= \pm \frac{1}{2}\right)=0, \quad$ and $\quad \hat{v}\left(\hat{r}, \hat{z}= \pm \frac{1}{2}\right)= \pm \hat{r}$.

Large aspect ratios: $\Gamma \gg 1$. We note that in the limit of large aspect ratios, that is as $\Gamma \rightarrow \infty$, the equations are reduced to being parabolic in $\hat{r}$ if one assumes that $\Gamma^{-1} \frac{\partial}{\partial \hat{r}} \ll 1$. This approach is taken, for example, in the work of Brady and Durlofsky [9] and corresponds to assuming that no radial flow features exist with length scales comparable to the separation of the two disks. Such an assumption will be invalid in "square" regions near the axis and the edge of the disks, where $\hat{r}=\mathrm{O}(1 / \Gamma)$ or $\hat{r}=1-\mathrm{O}(1 / \Gamma)$ (corresponding to $r^{*}=\mathrm{O}(h)$ or $\left.r^{*}=L-\mathrm{O}(h)\right)$. In these regions, the effects of radial viscous stresses must be included. Brady and Durlofsky imposed boundary conditions on the parabolic system based on ad hoc assumptions about the end regions; the reader is directed to their paper for specifics. We shall discuss this assumption that $\Gamma^{-1} \frac{\partial}{\partial \hat{r}} \ll 1$ in more detail when our global numerical results are presented.

\subsection{Self-similar flows in infinite domains}

If the flow domain is infinite in its radial extent, then Eqs. 1, 2 admit self-similar solutions of von Kármán form:

$\hat{u}=-\frac{1}{2} \hat{r} F^{\prime}(\hat{z}), \quad \hat{v}=\hat{r} G(\hat{z}), \quad \hat{w}=F(\hat{z}), \quad \hat{p}=\frac{1}{2} \hat{r}^{2} \Gamma^{2} K+Q(\hat{z})$,

where $K$ is a constant and a prime denotes differentiation with respect to the remaining independent variable, $\hat{z}$. The chosen form exactly satisfies the continuity equation (2) and ensures that the radial and transverse-pressure-gradient terms remain in the momentum equations.

Equation 1c decouples from the system and may be integrated once to determine the vertical pressure variation,

$Q(\hat{z})=F^{\prime}(\hat{z})-\operatorname{Re} \frac{1}{2} F^{2}(\hat{z})$.

After differentiation of Eq. 1a to eliminate the constant radial pressure gradient, $K$, the system reduces to two coupled ordinary differential equations

$G^{\prime \prime}=\operatorname{Re}\left(F G^{\prime}-F^{\prime} G\right)$,

$F^{(i v)}=\operatorname{Re}\left(F F^{\prime \prime \prime}+4 G G^{\prime}\right)$.

The boundary conditions are $F( \pm 1 / 2)=F^{\prime}( \pm 1 / 2)=0$ and $G( \pm 1 / 2)= \pm 1$. The system exhibits a pitchfork bifurcation at a critical $\mathrm{Re}=\mathrm{Re}_{\mathrm{c}} \approx 120$; see Fig. 1 .

These equations are said to be exact because none of the terms in the original Navier-Stokes equations have been neglected. We note that the terms of $\mathrm{O}\left(1 / \Gamma^{2}\right)$ in Eqs. 1a-c are identically zero when using the scalings (4). Hence, solutions of the Eqs. $6 \mathrm{a}, \mathrm{b}$ are also solutions of the original system in the limit, $\Gamma \rightarrow \infty$.

The system $(6 a, b)$ has been widely studied and many solutions have been found in the two-dimensional parameter space spanned by the Reynolds number and a measure of the relative rotation rates of the two disks; see [2]. For the purposes of this work, however, we restrict attention to the case of exact counter-rotation for Reynolds numbers in the range $\mathrm{Re} \in[0,500]$.

\subsection{Numerical solution in a finite domain}

When the flow domain is finite in its radial extent, we solve the axisymmetric Navier-Stokes equations using a Galerkin finite-element method. Standard continuation methods are used to track the resulting 

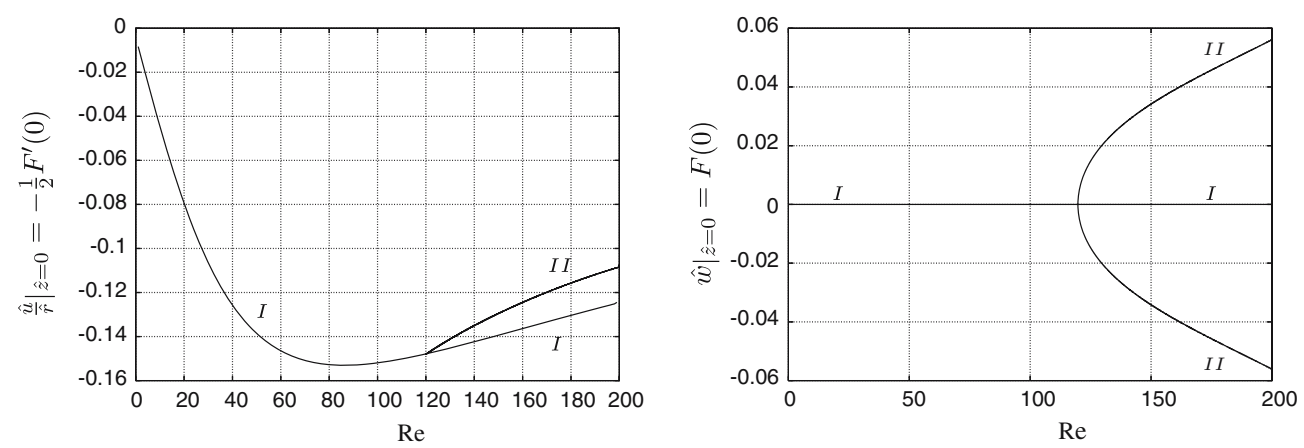

Fig. 1 The pitchfork bifurcation at $\mathrm{Re} \approx 120$ in the similarity solution described by (6a) and (6b). Note that the two branches of solution type $I I$ are interchanged by reflection in the midplane $z=0$, so that both branches have the same radial flow measure $(\hat{u} / \hat{r})$ but the transverse flow $(\hat{w})$ is inverted

solutions in the two-dimensional parameter space spanned by the Reynolds number, Re, and the aspect ratio of the domain, $\Gamma$.

The problem is formulated in the unscaled dimensionless coordinates, $(r, z)$, defined in Sect. 2, in which case $\Gamma \equiv 1$ in the governing equations $(1,2)$ and we drop the caret notation. The cylindrical domain is decomposed into isoparametric, axisymmetric $Q_{2} P_{-1}$ finite elements; see [13, p. 554] for example. The pressure is discontinuous across element boundaries and is approximated by piecewise bi-linear polynomials, $\xi_{j}, p=\sum_{j} p_{j} \xi_{j}$, where $p_{j}$ are the discrete pressure unknowns. The two global coordinates $(r, z)$ and the three velocity components $(u, v, w)$ are continuous across element boundaries and are approximated by piecewise bi-quadratic polynomials, $\phi_{i}$, e.g., $u=\sum_{i} u_{i} \phi_{i}$, where $u_{i}$ are the velocity unknowns.

The finite-element method is used to calculate approximate solutions to the weak form of the NavierStokes equations, obtained by weighting $(1,2)$ by suitable test functions and integrating over the fluid domain. A consequence of the assumption of axisymmetric flow is that we can integrate over the azimuthal coordinate, $\theta$, analytically and cancel the resulting factors of $2 \pi$.

In a Galerkin formulation, the test functions are the same as the functions used to interpolate the unknowns; hence, the momentum equations are weighted by the velocity basis functions, $\phi_{i}$. The viscous and pressure terms are integrated by parts, ensuring that the highest derivatives in the equations are firstorder only. An application of the divergence theorem yields the desired weak form, and leads to a set of coupled, nonlinear residual equations:

$$
\begin{aligned}
\mathcal{U}_{i}= & \int_{-\frac{1}{2}}^{\frac{1}{2}} \int_{0}^{\Gamma}\left[\operatorname{Re}\left(u \frac{\partial u}{\partial r}+w \frac{\partial u}{\partial z}-\frac{v^{2}}{r}\right)-\frac{p}{r}+(1+\gamma) \frac{u}{r^{2}}\right] \phi_{i} r \mathrm{~d} r \mathrm{~d} z \\
& +\int_{-\frac{1}{2}}^{\frac{1}{2}} \int_{0}^{\Gamma}\left[\left(-p+(1+\gamma) \frac{\partial u}{\partial r}\right) \frac{\partial \phi_{i}}{\partial r}+\left(\frac{\partial u}{\partial z}+\gamma \frac{\partial w}{\partial r}\right) \frac{\partial \phi_{i}}{\partial z}\right] r \mathrm{~d} r \mathrm{~d} z \\
& -\Gamma \int_{-\frac{1}{2}}^{\frac{1}{2}}\left[-p+(1+\gamma) \frac{\partial u}{\partial r}\right]_{r=\Gamma} \phi_{i} \mathrm{~d} z, \\
\mathcal{V}_{i}= & \int_{-\frac{1}{2}}^{\frac{1}{2}} \int_{0}^{\Gamma}\left[\operatorname{Re}\left(u \frac{\partial v}{\partial r}+w \frac{\partial v}{\partial z}+\frac{u v}{r}\right)+\frac{v}{r^{2}}-\gamma \frac{1}{r} \frac{\partial v}{\partial r}\right] \phi_{i} r \mathrm{~d} r \mathrm{~d} z \\
& +\int_{-\frac{1}{2}}^{\frac{1}{2}} \int_{0}^{\Gamma}\left[\left(\frac{\partial v}{\partial r}-\gamma \frac{v}{r}\right) \frac{\partial \phi_{i}}{\partial r}+\frac{\partial v}{\partial z} \frac{\partial \phi_{i}}{\partial z}\right] r \mathrm{~d} r \mathrm{~d} z-\Gamma \int_{-\frac{1}{2}}^{\frac{1}{2}}\left[\frac{\partial v}{\partial r}-\gamma \frac{v}{\Gamma}\right]_{r=\Gamma} \phi_{i} \mathrm{~d} z,
\end{aligned}
$$




$$
\begin{aligned}
\mathcal{W}_{i}= & \int_{-\frac{1}{2}}^{\frac{1}{2}} \int_{0}^{\Gamma}\left[\operatorname{Re}\left(u \frac{\partial w}{\partial r}+w \frac{\partial w}{\partial z}\right)\right] \phi_{i} r \mathrm{~d} r \mathrm{~d} z \\
& +\int_{-\frac{1}{2}}^{\frac{1}{2}} \int_{0}^{\Gamma}\left[\left(\frac{\partial w}{\partial r}+\gamma \frac{\partial u}{\partial z}\right) \frac{\partial \phi_{i}}{\partial r}+\left(-p+(1+\gamma) \frac{\partial w}{\partial z}\right) \frac{\partial \phi_{i}}{\partial z}\right] r \mathrm{~d} r \mathrm{~d} z \\
& -\Gamma \int_{-\frac{1}{2}}^{\frac{1}{2}}\left[\frac{\partial w}{\partial r}+\gamma \frac{\partial u}{\partial z}\right]_{r=\Gamma} \phi_{i} \mathrm{~d} z .
\end{aligned}
$$

The parameter $\gamma$ is used to specify whether the equations are solved in conventional $(\gamma=0)$ or stressdivergence form $(\gamma=1)$. In the latter case, the "natural" boundary conditions are that the fluid traction is zero; in the case $\gamma=0$, the corresponding boundary conditions are that the pseudo-traction is zero, see (11) for the distinction between traction and pseudo-traction.

The surface integrals over the upper and lower walls are omitted because we impose Dirichlet conditions along these boundaries. The appropriate no-slip boundary conditions are $u=w=0$ on $z= \pm 1 / 2$ and, for exact counter-rotation, we impose $v=-r$ on $z=-1 / 2$ and $v=r$ on $z=1 / 2$. The boundary conditions at the axis, $r=0$, are $u=0, v=0$ and $\partial w / \partial r=0$. This last condition is the "natural" boundary condition of (7c) even when $\gamma=1$, because the Dirichlet condition on $u$ implies that $\partial u / \partial z=0$ along the axis. This condition is implicitly enforced in our formulation by the omission of the "surface" integral along the line $r=0$ in $(7 \mathrm{c})$.

Finally, the weak form of the continuity equation is obtained by weighting it with the pressure basis functions, $\xi_{j}$, and integrating over the volume,

$\mathcal{P}_{j}=\int_{-\frac{1}{2}}^{\frac{1}{2}} \int_{0}^{\Gamma}\left[\frac{1}{r} \frac{\partial}{\partial r}(r u)+\frac{\partial w}{\partial z}\right] \xi_{j} r \mathrm{~d} r \mathrm{~d} z$.

The nonlinear system of equations $\left(\mathcal{U}_{i}, \mathcal{V}_{i}, \mathcal{W}_{i}, \mathcal{P}_{j}\right)^{T}=0$ is then assembled and solved numerically via a Newton method.

It remains only to specify the boundary condition at the end of the domain, $r=\Gamma$. We find that this boundary condition can have a profound effect on the behaviour of the solution and consider three main alternatives.

\subsubsection{Closed-end boundary conditions}

In many experimental configurations, the disks are enclosed by a fixed impermeable end wall. The required boundary conditions in this case are that $u=v=w=0$ on the edge $r=\Gamma$; and we obtain the same results for $\gamma=0$ and $\gamma=1$. The condition $v=0$ induces a discontinuity in the swirl velocity $v$ at the corners $r=\Gamma, z= \pm 1 / 2$ because the upper/lower boundaries impose $v= \pm \Gamma$ at these points. In contrast to the well-known driven-cavity problem, there is no associated pressure singularity because the pressure does not feature in the azimuthal momentum equation. Nonetheless, we have taken great care to obtain results that are independent of the treatment of the corner regions. Following the methods successfully employed in Taylor-Couette flow computations (see for example [14]), a simple approach is to reduce the swirl velocity of the disks to zero smoothly, but rapidly, with a corner refinement of the computational mesh in these regions. Numerical results are then seen to be independent of the length scale over which this smoothing is applied (for sufficiently small length scales). For sufficiently fine grids, the results are identical to those obtained when the corner discontinuities are explicitly included in the approximation for the swirl velocity, $v=V_{\text {discont }}+\sum_{i} v_{i} \phi_{i}$. In practice, of course, in any experimental investigation there will be a small gap between the disks and any surrounding shroud. 


\subsubsection{Open-end boundary conditions}

The earliest numerical studies of flow between finite rotating disks were motivated by the problem of flows in differentially rotating cylindrical drops [15]. The boundary conditions chosen in such studies were appropriate for a 'free surface' confined to the line $r=\Gamma$. Specifically, the velocity normal to the surface is zero, $u=0$, and the tangential fluid traction is also zero. The traction conditions are enforced by omitting the surface integrals in (7b) and (7c) and setting $\gamma=1$. We use these boundary conditions in order to compare our results with the recent paper of Witkowski et al. [12]). In addition, we briefly consider traction-free boundary conditions, the "natural" boundary conditions when $\gamma=1$; and pseudo-traction-free boundary conditions, the "natural" boundary conditions when $\gamma=0$. In both cases, the normal fluid traction or pseudo-traction, rather than the normal fluid velocity, is set to zero.

\subsubsection{Boundary conditions consistent with the similarity solution}

We expect to be able to resolve the similarity solution throughout the entire finite domain, provided that the condition applied at $r=\Gamma$ does not preclude the similarity form. One possibility is to impose the similarity solution as a Dirchlet condition. The similarity solution, $u_{\mathrm{ss}}=\left(r u_{\mathrm{ss}}, r v_{\mathrm{ss}}, w_{\mathrm{ss}}\right)^{T}$, is obtained by solving $(6 \mathrm{a}, 6 \mathrm{~b})$; here we use $u_{\mathrm{ss}}(z), v_{\mathrm{ss}}(z)$ and $w_{\mathrm{ss}}(z)$ to denote the quantities $-F^{\prime}(z) / 2, G(z)$ and $F(z)$, respectively. This solution can then be imposed at every node on the boundary by setting

$u(r=\Gamma, z)=\Gamma u_{\mathrm{ss}}(z)-\Gamma \int_{-\frac{1}{2}}^{\frac{1}{2}} u_{\mathrm{ss}}(z) \mathrm{d} z$,

$v(r=\Gamma, z)=\Gamma v_{\mathrm{ss}}(z), \quad w(r=\Gamma, z)=w_{\mathrm{ss}}(z)$.

The inclusion of the integral term here ensures that conservation of mass is achieved in the discretised problem by subtracting the mass flux per unit length into the domain from the radial velocity component. These boundary conditions do indeed resolve the similarity solution throughout the entire domain, although for coarse meshes the discrete-mass-conservation requirement introduces a small correction region near $r=\Gamma$.

Once $\mathrm{Re}>\mathrm{Re}_{\mathrm{c}} \approx 120$, however, the solution for $u_{\mathrm{ss}}$ is non-unique and one of the multiple steady solutions must be selected and imposed at the edge. In fact, the selected solution is resolved throughout the domain, but remains stable to temporal perturbations, even when the imposed condition is an unstable solution of the similarity equations. Thus, the Dirichlet conditions are too restrictive (or "too hard"), in the sense that the reflectional symmetry about the mid-plane will not be broken in the finite domain if that symmetry is enforced via the edge condition (even though the condition is applied only along a line within the domain and the other points in the domain are free to become asymmetric).

A more elegant boundary condition (that does not impose a symmetry) may be formulated by requiring that the traction at the location $r=\Gamma$ is consistent with the form of the similarity solution. In general, the similarity form (4) can be made unsteady, giving a solution in the form

$u=r u_{\mathrm{ss}}(z, t), \quad v=r v_{\mathrm{ss}}(z, t), \quad w=w_{\mathrm{ss}}(z, t), \quad p=\frac{1}{2} r^{2} K(t)+Q(z, t)$.

A consequence of the addition of the acceleration term is that Eq. 5 becomes

$Q(z, t)=w_{\mathrm{ss}}^{\prime}(z, t)-\operatorname{Re}\left(\frac{1}{2} w_{\mathrm{ss}}^{2}(z, t)+\int^{z} \frac{\partial w_{\mathrm{ss}}}{\partial t}(s, t) \mathrm{d} s\right)$.

The fluid-traction components normal to the boundary $r=\Gamma$ are

$\sigma_{u}=-p+(1+\gamma) \frac{\partial u}{\partial r}, \quad \sigma_{v}=\frac{\partial v}{\partial r}-\gamma \frac{v}{r}, \quad \sigma_{w}=\frac{\partial w}{\partial r}+\gamma \frac{\partial u}{\partial z}$. 
Thus, using the scalings (9) and Eq. 10 shows that the following tractions are consistent with the similarity solution:

$$
\begin{aligned}
\sigma_{u} & =-\frac{1}{2} r^{2} K(t)+\operatorname{Re}\left(\frac{1}{2} w_{\mathrm{ss}}^{2}(z, t)+\int^{z} \frac{\partial w_{\mathrm{ss}}}{\partial t}(s, t) \mathrm{d} s\right)+(3+\gamma) u_{\mathrm{ss}}(z, t), \\
\sigma_{v} & =(1-\gamma) v_{\mathrm{ss}}(z, t), \\
\sigma_{w} & =\gamma r \frac{\partial u_{\mathrm{ss}}}{\partial z}(z, t),
\end{aligned}
$$

where the continuity equation has been used to replace $\partial w_{\mathrm{ss}} / \partial z$ by $-2 u_{\mathrm{ss}}$. Without any loss of generality we can insist that the pressure constant is zero at the end of the domain, $K=0$, and then require that the velocity field of the full solution is the similarity solution at the edge of the domain, i.e. $u_{\mathrm{ss}}=u / \Gamma$, $v_{\mathrm{ss}}=v / \Gamma$ and $w_{\mathrm{ss}}=w$ at $r=\Gamma$. The resulting traction conditions then become

$$
\begin{aligned}
\sigma_{u} & =\operatorname{Re}\left[\frac{1}{2} w^{2}+\left.\int^{z} \frac{\partial w}{\partial t}(s, t)\right|_{r=\Gamma} \mathrm{d} s\right]+(3+\gamma) \frac{u}{\Gamma}, \\
\sigma_{v} & =(1-\gamma) \frac{v}{\Gamma}, \\
\sigma_{w} & =\gamma \frac{\partial u}{\partial z},
\end{aligned}
$$

and this is easily imposed (directly) via the surface-integral terms in the weak form of the momentum equations. We note that, for steady problems, the integral term will be zero.

\section{Results}

Two different implementations of the same problem have been developed, using separate libraries for the solution of elliptic systems via the finite-element method, namely oomph-lib [16] and ENTWIFE [17]. The results (as shown in this section) of both formulations agree to within the specified tolerance of the methods, which is typically that the maximum residual is below $10^{-8}$.

\subsection{The symmetric states}

The similarity solution may be interpreted as a near-axis expansion in the radial coordinate, and so we expect it to be at its most accurate (compared to a finite-domain solution) in a central region near $r=0$. If the boundary conditions at $r=\Gamma$ are not compatible with the similarity solution, then we might hope that this influence will be confined to a zone near the end of the domain in which the solution adjusts to the enforced boundary conditions.

We first consider the closed-end boundary condition, corresponding to the disks being surrounded by a fixed, impermeable shrouding cylinder. Results for the vertical velocity at a fixed height, $w(r, z=-1 / 4)$, are shown in Fig. 2 together with the corresponding similarity solutions in a domain of aspect ratio $\Gamma=10$. Here we choose a measure of the $w$-velocity component at $z=-1 / 4$ in order to obtain a non-zero measure of symmetric states; it is worth noting that this is an arbitrary choice and any metric displays similar behaviour.

We find, in agreement with [9], that the similarity solution provides an accurate quantitative prediction for the flow in a region near the axis of rotation, and that, for sufficiently large aspect ratios, $\Gamma \geq 10$, the region always occupies the same proportion of the domain at a given Re, i.e., the results are independent of $\Gamma$ when presented as functions of the scaled radial coordinate, $\hat{r}$. The axial extent of the region decreases as Re increases and Brady and Durlofsky [9] showed that the region vanishes for sufficiently large values 


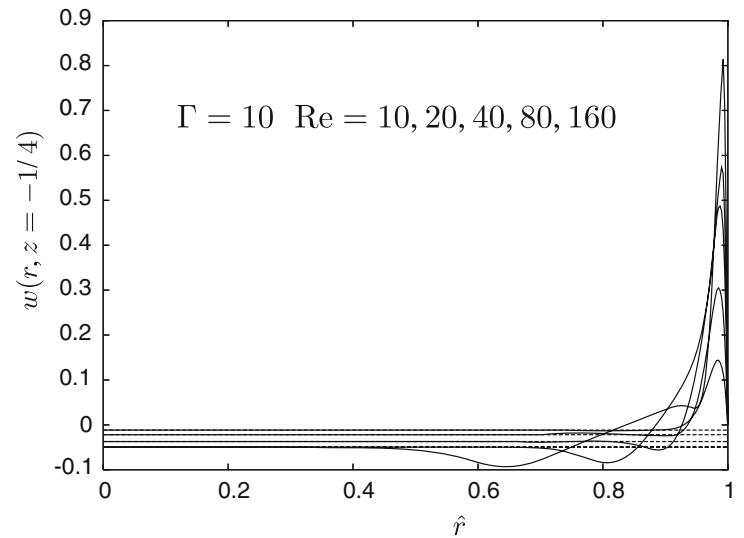

Fig. 2 Vertical velocity, $w$, as a function of the scaled radial coordinate $\hat{r}=r / \Gamma$ at a fixed height, $z=-1 / 4$, for the case $\Gamma=10$. Solutions obtained in the finite domain with closed-end boundary conditions (solid lines) are compared to the similarity solution (dashed lines) from $\mathrm{Re}=10$ (top) to $\operatorname{Re}=160$ (bottom). Note that $w$ is independent of $\hat{r}$ for the similarity solution

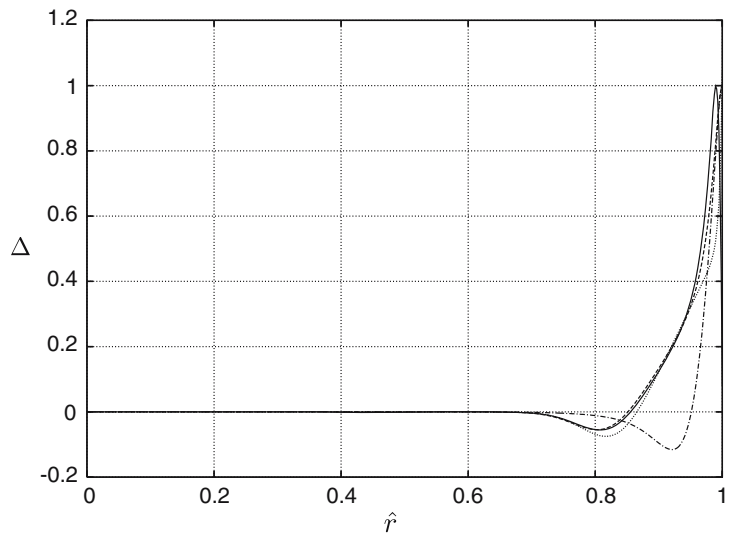

Fig. 3 Scaled deviation of the velocity from the similarity solution, $\Delta=\left(w-w_{\mathrm{ss}}\right) / \max _{\hat{r}}\left(w-w_{\mathrm{ss}}\right)$, as a function of the scaled radial coordinate $\hat{r}=r / \Gamma$ at a fixed height, $z=-1 / 4$, for the case $\Gamma=10, \operatorname{Re}=80$. Solutions are shown for four different boundary conditions at $r=\Gamma$ : closed-end (solid line), open-end (dashed line), traction-free (dotted line) and pseudo-traction-free (dot-dashed line). In each case deviation from the similarity solution is first observed at $\hat{r} \approx 0.7$

of Re in arbitrarily large (but still finite) domains. At such high values of Re, however, such results are of less interest if the symmetric state becomes unstable via bifurcations at lower Reynolds numbers.

We find the same general behaviour for a number of alternative boundary conditions applied at $r=\Gamma$ and, in fact, the size of the adjustment zone does not vary greatly between the different cases. A comparison between four different boundary conditions at $\Gamma=10$ and $\mathrm{Re}=80$, is shown in Fig. 3. The accuracy of the similarity solution is indicated by plotting the deviation of the vertical velocity from the similarity solution at a fixed height, $w(r, z=-1 / 4)-w_{\mathrm{ss}}(r, z=-1 / 4)$. In order to compare the different boundary conditions, the measure is scaled so that the maximum deviation in the range $r \in[0, \Gamma]$ is always one. In fact, the absolute magnitude of the deviation is only significantly different in the case of the pseudo-traction-free boundary condition, when it is an order of magnitude smaller than the other three cases.

We conclude, therefore, that, provided the flow remains symmetric, the similarity solution provides a good representation of the solution over a sizeable portion of the finite domain for moderate Reynolds numbers $(\mathrm{Re} \lesssim 200)$ irrespective of the boundary conditions.

\subsection{Midplane-symmetry breaking}

The similarity solution loses midplane symmetry via a supercritical pitchfork bifurcation at $\operatorname{Re}=\operatorname{Re}_{\mathrm{c}} \approx$ 120, see Sect. 2.1. This pitchfork bifurcation is independent of $\Gamma$ and is exactly reproduced by the full elliptic system when fluid tractions consistent with the form of the similarity solution (Eqs. 12a,c), are applied at $r=\Gamma$. Using these end conditions, we find $\mathrm{Re}_{\mathrm{c}}=119.78$ at a mesh resolution of $150 \times 30$ elements, in agreement with the results from the system $(6 a, b)$. The accurate resolution of the bifurcation confirms that these boundary conditions do indeed faithfully reproduce the behaviour of the similarity solution over the whole domain. We should note, however, that these boundary conditions do not preclude the co-existence of more complex, non-self-similar states.

In the finite domain, we would not expect the symmetry-breaking bifurcation to be suppressed for general boundary conditions. It remains to be seen, however, whether the bifurcation in the similarity 
solution has any bearing on the finite-domain problem. Brady and Durlofsky [9] solved the parabolic system obtained from the large-aspect-ratio limit $\Gamma \rightarrow \infty$ of the Navier-Stokes equations $(1,2)$, but were unable to find non-uniqueness in their solution. In contrast, Harriot and Brown [15] solved the problem using the open-end boundary conditions and found a midplane-symmetry breaking pitchfork bifurcation at $\operatorname{Re}_{\mathrm{c}} \approx 109$ when $\Gamma=1$. In addition, the recent work of Nore et al. [11] found axisymmetric pitchfork bifurcations for closed-end conditions when $\Gamma \leq 2$. In that regime, however, bifurcations to non-axisymmetric states were found to occur at lower Reynolds numbers than the midplane-symmetry breaking bifurcations; we shall discuss this in our concluding section.

Initially, we demonstrate convergence of our numerical results, by locating the first midplane-symmetry breaking pitchfork bifurcation, $\mathrm{Re}_{\mathrm{p}}$, for closed-end boundary conditions when $\Gamma=10$. The results for a number of different (uniform) mesh resolutions in the half domain $(r, z) \in[0, \Gamma] \times[0,1 / 2]$ are shown in Table 1 . The location of the bifurcation is well-resolved by a uniform mesh of $150 \times 30$ elements in the complete domain and this resolution was used for the majority of presented computations. Convergence of selected results was confirmed by repeating the calculations at a higher resolution. We note that the actual number of elements over the whole domain is somewhat greater than $2 N_{r} \times N_{z}$, owing to five levels of corner refinement employed near $r=\Gamma$.

Table 2 shows the location of the first pitchfork bifurcation when $\Gamma=10$ for a number of different boundary conditions. Although the region of validity of the symmetric similarity solution appears to be independent of the boundary conditions, the symmetry-breaking bifurcation is significantly influenced by the boundaries. In no case, however, does the location of the bifurcation coincide with the symmetrybreaking bifurcation of the similarity solution; the closed-end is the closest at $\operatorname{Re} \approx 108$. It is possible to perform homotopy continuation to demonstrate that the similarity-solution bifurcation is connected to the corresponding pitchfork bifurcation in a two-dimensional parameter space spanned by the Reynolds number and a homotopy parameter for the closed-end and traction-free boundary conditions. For the openend boundary condition, the connection is via a path in a three-dimensional parameter space spanned by the Reynolds number, the aspect ratio and the homotopy parameter.

Table 1 The location of the pitchfork bifurcation at $\Gamma=10$ for a uniform mesh of $N_{r} \times N_{z}$ elements over the half domain with closed-end conditions, $u=v=w=0$ at $r=\Gamma$, corresponding to a fixed impermeable shrouding cylinder

\begin{tabular}{rrll}
\hline$N_{r}$ & $N_{z}$ & $\operatorname{Re}_{\mathrm{p}}(\Gamma=10)$ & \% change \\
\hline 50 & 5 & 110.055 & - \\
100 & 10 & 108.647 & $1.30 \%$ \\
150 & 15 & 108.405 & $0.22 \%$ \\
200 & 20 & 108.342 & $0.06 \%$ \\
\hline
\end{tabular}

Table 2 The location of the pitchfork bifurcation at $\Gamma=10$ for a uniform mesh of $150 \times 15$ elements over the half domain under different boundary conditions at $r=\Gamma$ : closed end, $u=v=w=0$; open end, $\gamma=1, u=0$ and the tangential traction is zero; traction free, $\gamma=1$ and all components of the fluid traction are zero; pseudo-traction free, $\gamma=0$ and all components of the fluid traction are zero

\begin{tabular}{lr}
\hline Boundary condition & $\operatorname{Re}_{\mathrm{p}}(\Gamma=10)$ \\
\hline Similarity solution & 119.8 \\
Closed end & 108.4 \\
Open end & 26.7 \\
Traction free & 39.4 \\
Pseudo-traction free & 217.3
\end{tabular}

The location of the bifurcation is strongly dependent on the boundary conditions, despite the fact that the symmetric base state is well approximated by the similarity solution in all cases. Here we also show the critical Reynolds number for the similarity solution, although this is obviously independent of $\Gamma$ 
In general, the Reynolds number at which the first pitchfork bifurcation occurs will be a function of the aspect ratio, $\operatorname{Re}=\operatorname{Re}_{\mathrm{p}}(\Gamma)$. At least for the closed-end condition, one might anticipate that the bifurcation in the finite domain, at $\mathrm{Re} \approx 108$, is closely related to the bifurcation in the similarity solution at $\operatorname{Re} \approx 120$. It would be natural to expect (naively) that $\operatorname{Re}_{\mathrm{p}}(\Gamma)$ approaches 120 as $\Gamma$ increases. We find that this is not the case. Moreover, the asymmetric bifurcated states show no agreement with the corresponding asymmetric self-similar solution. For the closed-end boundary conditions, Fig. 4 presents a comparison of $w(r=0, z=-1 / 4)$ for both the bifurcating similarity solution (solid line) and the bifurcating global solution (data points and dashed line). As we have noted previously, there is excellent agreement between the symmetric state (branch $I$ ) and the full solution (over the full range of Re here), but there is clearly no quantitative agreement for the bifurcating states (branch $I I$ ).

In fact, there is no qualitative agreement between the bifurcated states of the finite-domain problem and those of the self-similar solution. The reason that non-uniqueness is absent in [9] was the limiting assumption that

$\frac{1}{\Gamma} \frac{\partial}{\partial \hat{r}} \ll 1 \quad$ as $\Gamma \rightarrow \infty$

Our numerical results indicate that, in contrast to the similarity solution, the loss of midplane symmetry in the finite domain is associated with structures that have a radial length scale comparable to the separation of the two disks; see Figs. 8 and 9. Any attempt to consider a parabolic interior flow based on the above scaling, will necessarily eliminate such states.

\subsubsection{Closed-end conditions}

For the experimentally motivated, closed-end boundary conditions, Fig. 5 shows the locus of the pitchfork bifurcation and the locus of saddle-node boundaries in the two-dimensional parameter space spanned by the Reynolds number and the aspect ratio. The recent data presented by Nore et al. [11], who considered the small aspect ratio limit $(\Gamma \leq 2)$, is included for additional validation and is found to be in excellent agreement with our results. We note in passing that their analysis uses the disk radius as the natural length-scale, leading to different definitions of the aspect ratio and Reynolds number.

To aid in the interpretation of Fig. 5, we also provide a schematic of the bifurcation structure (in Fig. 6) for increasing values of the aspect ratio. Each bifurcation diagram (a)-(l) shows only half of the full dia-

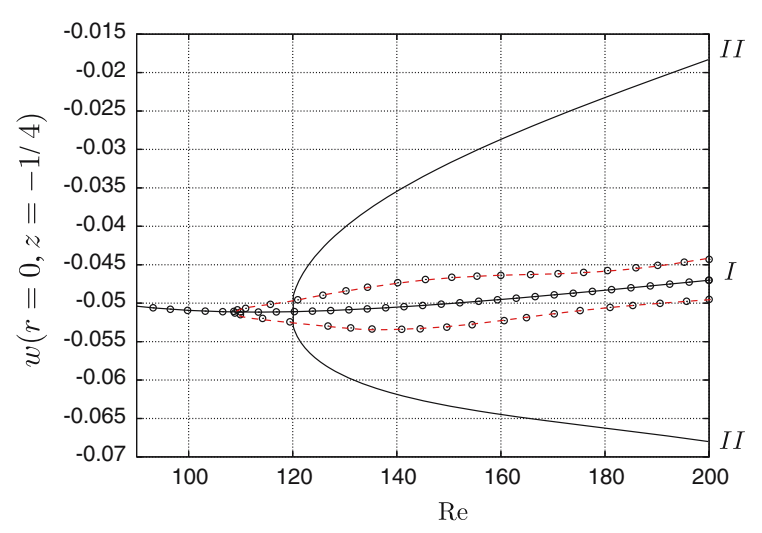

Fig. 4 A bifurcation diagram for $\Gamma=10$. The solid lines show the pitchfork bifurcation in the self-similar solution, whilst the data points and dashed lines show the finite-disk solution with a fixed shrouding cylinder at $r=\Gamma$. The solution branches $I, I I$ are as marked in Fig. 1

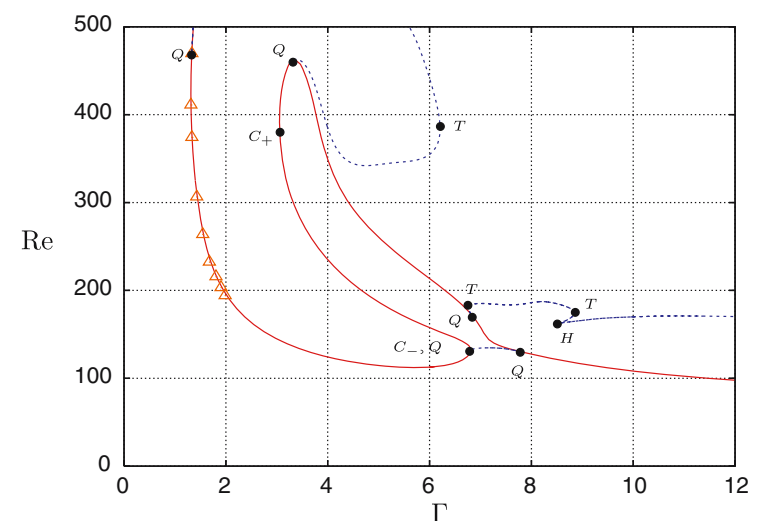

Fig. 5 The locus of the primary pitchfork bifurcation (solid line) and the locus of saddle-node points (dashed lines) for the closed-end boundary condition. The data points $(\triangle)$ are obtained from the work of Nore et al. [11]. Here $C_{+}, C_{-}$ denote coalescence points, $T$ transcritical points, $H$ a hysteresis point and $Q$ quartic points 
gram, since each is symmetric about the horizontal axis. Quantitative bifurcation diagrams showing the sequence (a)-(1) have been generated, but they show the topology of the solutions with less clarity than this schematic sequence.

The cross section (a) corresponds to low values of $\Gamma$ for which the first bifurcation is supercritical and the second bifurcation is subcritical. This is not evident in Fig. 5 because the schematic diagram exaggerates the separation of the saddle node and second bifurcation point. The sequence (b)-(l) corresponds to increasing $\Gamma$ towards $\Gamma=12$, the upper limit in Fig. 5. In particular, we note the existence of the three transcritical points at critical aspect ratios between Figs. (e) and (f), (f) and (g) and (k) and (l); these transcritical points are located at $\Gamma \approx 6.21,6.77$ and 8.85 , respectively. There is also a hysteresis point at $\Gamma \approx 8.51$, shown by the transition from diagram $(\mathrm{j})$ to $(\mathrm{k})$.

At these moderate to large aspect ratios, therefore, the behaviour of the system is extremely complex and completely different to that of the similarity solution.

\subsubsection{Open-end conditions}

The case of open-end boundary conditions has been studied in [12] for large-aspect-ratio counterrotating-disk flow. They considered only the linear stability problem, however, and do not present details of the bifurcation structure or the nonlinear, bifurcated states. Witkowski et al. found that for this choice of end condition, a pitchfork bifurcation is found at low to moderate values of $\Gamma$, but is replaced by a Hopf bifurcation at larger values of $\Gamma$. A parallel-flow approximation applied to the base flow followed by a temporal stability analysis to perturbations of normal mode form was found to be in agreement with the numerical results for large $\Gamma$. The main result of their paper was that the location of the Hopf bifurcation scales with the inverse square root of the aspect ratio, $\operatorname{Re}_{\mathrm{Hopf}} \sim \Gamma^{-1 / 2}$.

We extend the results of Witkowski et al. to determine the locus of the pitchfork bifurcation over a wider range of the parameter space spanned by the Reynolds number and the aspect ratio. The results are shown in Fig. 7 together with the data of Witkowski et al. The datasets differ when the first instability becomes a Hopf bifurcation. Once again, we find a complex behaviour that appears to be totally unrelated to the behaviour of the similarity solution.

(a)

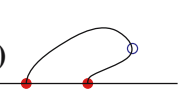

(e)

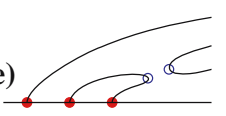

(b)

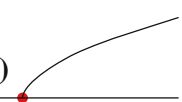

(c)

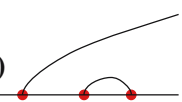

(d)

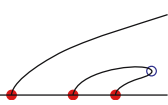

(f)

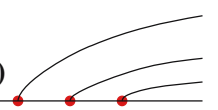

(g)

(h)
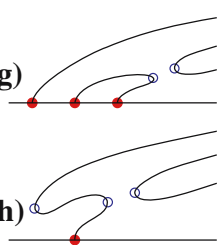

(j)
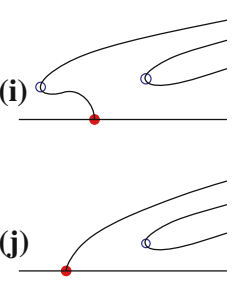

(k)

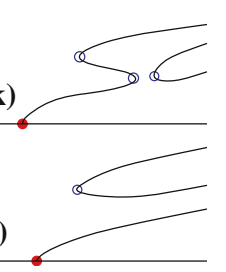

Fig. 6 A schematic diagram of the bifurcation sequence as a function of $\operatorname{Re} \in[0,500]$ for fixed values of $\Gamma$. The diagrams (a)-(I) correspond to aspect ratios: $\Gamma \approx$ $1.4,2.5,3.15,4,6,6.5,6.79,6.81,7,8,8.75,12$. The locus of the saddle-node points (o) are shown as the dashed lines in Fig. 5 , whereas pitchfork bifurcations $(\bullet)$ are shown as the solid line in that figure

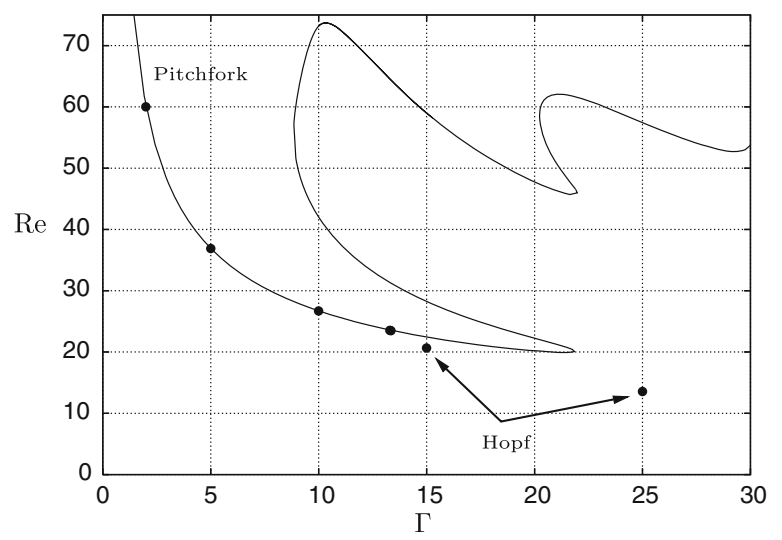

Fig. 7 The instability boundaries for an open-end condition applied at $r=\Gamma$. The data points denote the results of Witkowski et al. for the first instability threshold, whereas the solid line shows our results for the locus of the pitchfork bifurcation. This dataset should be compared to that shown in Fig. 5 (for a closed end) although we do not present the saddle-node boundaries in this case 


\subsection{Nonlinear stationary-wave states}

In this section, we briefly describe the nonlinear states that arise from the pitchfork bifurcation. A possible description of these states by asymptotic means is under investigation by the authors, and we restrict ourselves to presenting some of the main qualitative features here.

We first present results for the closed-end boundary conditions when $\Gamma=10$. Note that in the following discussion, we shall make use of the symmetry about the horizontal axis and restrict attention to the half of the bifurcation diagram shown in Fig. 6(1). The pitchfork bifurcation is located at $\operatorname{Re}_{\mathrm{p}} \approx 108.4$ and the saddle-node at $\mathrm{Re}_{\mathrm{s}} \approx 170.1$. Thus, when $\mathrm{Re}=150$, there are two possible solutions: the symmetric solution and the asymmetric bifurcated solution. Meridional streamlines and contours of the azimuthal velocity for these solutions are shown in Fig. 8. When $\mathrm{Re}=200$, however, there are four possible solutions: the symmetric solution, the asymmetric solution emanating from the pitchfork bifurcation and two isolated asymmetric solutions connected through the saddle-node bifurcation. Figure 9 shows meridional streamlines and contours of azimuthal velocity for the three asymmetric solutions; the symmetric solution does not differ greatly from the symmetric solution at $\operatorname{Re}=150$ and is not shown.

As we have noted in the previous section, the bifurcated states arise with radial length scales that are comparable to the disk separation. In particular, any metric of the solution as a function of radial location displays a wavelike behaviour. An obvious metric to choose is the meridional stream function $\psi(r, z)$ where $u=-\psi_{z} / r$ and $w=\psi_{r} / r$. For the von Kármán similarity solution it is straightforward to see that, in terms of our solution in Sect. 2.1, we have $\psi(r, z)=\frac{1}{2} r^{2} F(z)$. Although these finitedomain stationary-wave solutions are not of von Kármán form, we shall choose to scale the measure of the solution by the $r^{2}$ factor. In figure 10, we also choose to scale the measure by an additional factor $\log (r)$, although for the purposes of this limited description one can view that choice as being empirically motivated.
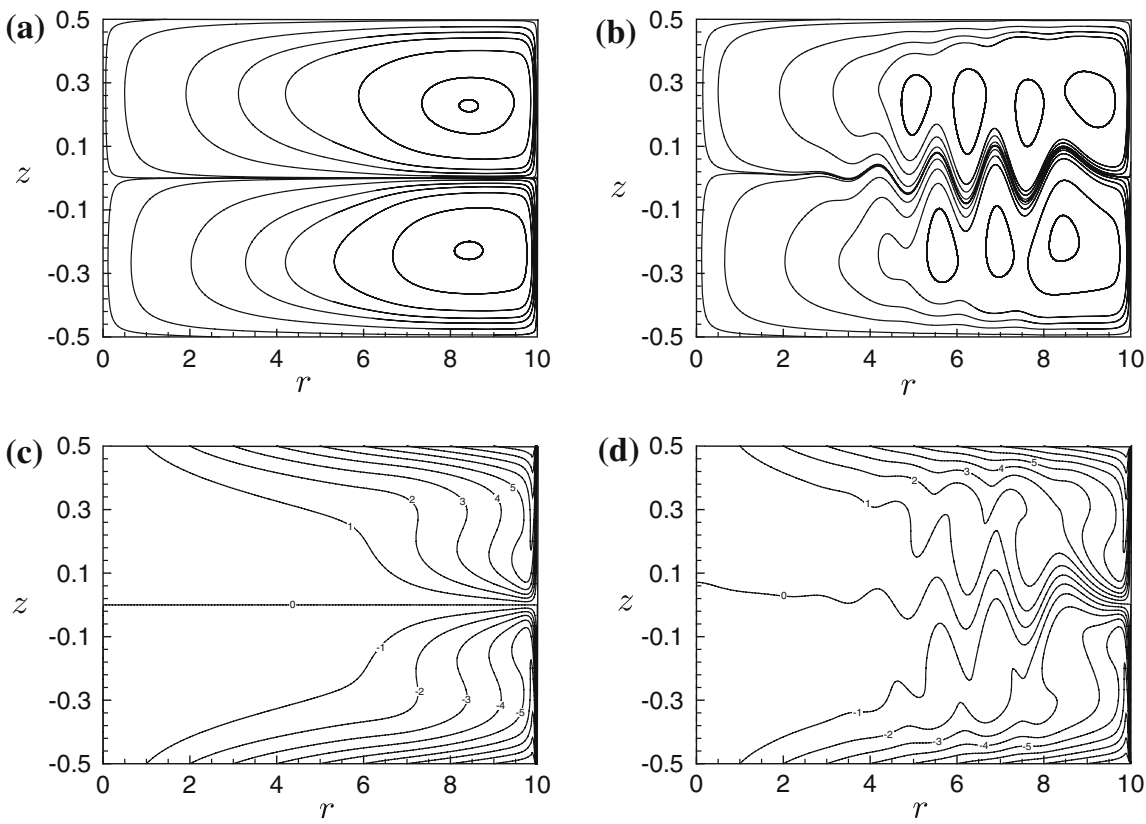

Fig. 8 Streamlines $(\mathbf{a}, \mathbf{b})$ and contours of azimuthal velocity, $v,(\mathbf{c}, \mathbf{d})$ for the closed-end boundary conditions when $\Gamma=10$ and $\operatorname{Re}=150$. The symmetric solution is shown on the left $(\mathbf{a}, \mathbf{c})$ and the asymmetric bifurcated solution is shown on the right $(\mathbf{b}, \mathbf{d})$. Note that a third possible solution is the reflection of the asymmetric solution $(\mathbf{b}, \mathbf{d})$ in the line $z=0$ 

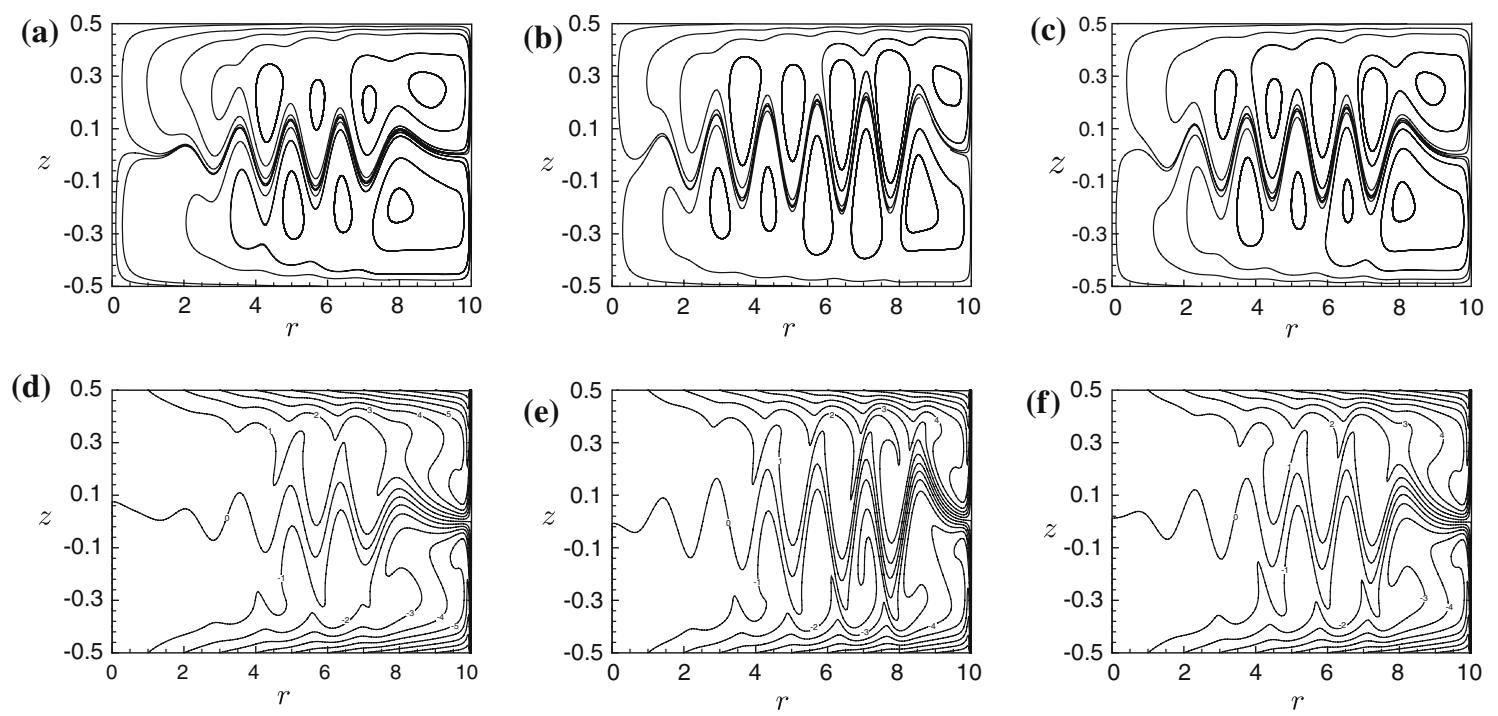

Fig. 9 Streamlines $(\mathbf{a}, \mathbf{b}, \mathbf{c})$ and contours of azimuthal velocity, $v,(\mathbf{d}, \mathbf{e}, \mathbf{f})$ for the closed-end boundary conditions when $\Gamma=10$ and $\mathrm{Re}=200$. There are seven possible solutions: the symmetric state, three asymmetric solutions and their reflections in the line $z=0$ (not shown here). (a, d) show the asymmetric solution on the branch connected to the pitchfork bifurcation; (b, e) and $(\mathbf{c}, \mathbf{f})$ show the solutions on the lower and upper branches of the isolated solution curve, respectively; see Fig. 6(1)

Fig. 10 The bifurcated, finite-domain solution with a closed-end condition at $\mathrm{Re}=150$ at a range of aspect ratios $\Gamma=10,15,20,25,30$.

Here we plot the scaled meridional stream function, $\psi$, as a function of the radial coordinate, along the midplane $(z=0)$ of the flow

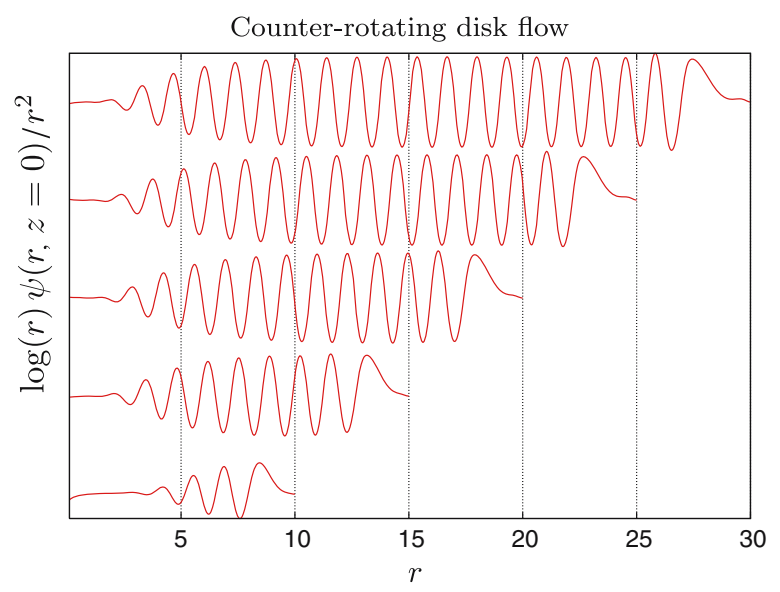

It is clear from Fig. 10 that the solutions are saturated wave states that have a well-defined wavelength. The wavelength (when non-dimensionalised on the gap-width) is independent of the aspect ratio of the domain, although there is some phase adjustment associated with the particular value of $\Gamma$ chosen.

Interestingly, the wave-state solutions appear to be generic solutions of the problem. The solutions have been observed for the open-end boundary conditions. Indeed, it is possible to perform a homotopy continuation to show that such states persist if the edge conditions are altered so that one imposes the asymmetric similarity solution (at the same Reynolds number) as a Dirichlet condition. In this sense, one might expect that these states are more general solutions of the unbounded flow and are not simply a consequence of the forcing at the edge of the finite domain. 


\section{Discussion}

In summary, we find the following answers to the three simple questions raised in the introduction:

(i) In general, the finite-domain problem does lose the midplane symmetry as the aspect ratio is increased. Continuation in a homotopy parameter demonstrates that each bifurcation is connected to the bifurcation of the similarity solution, but the path between the two in the space spanned by the Reynolds number, the aspect ratio and the homotopy parameter can be rather complex.

(ii) The bifurcation in the similarity solution is of no use in predicting the critical Reynolds number at which symmetry is lost in a finite-domain flow, even when $\Gamma$ is large. Over the range of aspect ratios studied here, $\Gamma \in[0,30]$, changing the boundary conditions causes vast differences in the critical parameters at which the pitchfork bifurcation is located. Thus, although the exact nature of the edge conditions has little influence on the symmetric solution, it is crucial in determining the bifurcation structure. For example, comparing Fig. 7 and 5, at $\Gamma=10$, we see that the critical Reynolds number differs by (approximately) a factor of four when switching the edge conditions from a closed end, to an open end. Moreover, neither case bears any resemblance to the similarity solution.

(iii) Despite the complete failure of the symmetry-broken similarity states (branch $I I$ ) to make useful predictions for a finite domain, the symmetric similarity solution (branch $I$ ) is an excellent predictor of the flow. For moderate Reynolds numbers, the agreement is good over sizeable portions of the domain and appears to be unchanged by the different edge constraints, all of which are nonlinear perturbations of the similarity solution.

The applicability of the symmetric similarity solution at low Reynolds numbers was exploited by Witkowski et al. to find a scaling for the first instability threshold as a function of aspect ratio. They assumed that the aspect ratio was large and made a parallel-flow approximation, after which they performed a linear stability analysis of the symmetric similarity solution. Although not explicitly stated in their paper, we believe that the scaling applies only to the Hopf bifurcation and find no obvious indication that it applies to our pitchfork bifurcation, at least in the range of $\Gamma$ investigated here.

There are two obvious points to be made here regarding our assumptions of a steady, axisymmetric solution. As shown in Fig. 7, there is always the possibility that the first instability is in fact a Hopf bifurcation leading to a time-dependent axisymmetric state. Although we have not performed any formal continuation of the Hopf boundaries, we have performed several unsteady computations to confirm our steady results and to assess the importance of time-dependent states. For the open-end boundary conditions, our unsteady results confirm the presence of the Hopf bifurcation predicted by Witkowski et al. but importantly, in Fig. 7 , there is a range of $\Gamma$ for which the first pitchfork only leads to instability of the symmetric state for a small range of $\mathrm{Re}$ before it is restabilised. Unsteady computations at $\mathrm{Re}=80$ and $\Gamma=10$ confirm that some of these restabilised regions are temporally stable, a feature that is missed in the results of Witkowski et al. Furthermore, for the closed-end condition, unsteady computations suggest that over the range of Re and $\Gamma$ presented in Fig. 5, there is no significant role played by a Hopf bifurcation.

A second possibility is that the first instability is non-axisymmetric and the work of Nore et al. [11] shows that non-axisymmetric instabilities are typically the first observed at low values of $\Gamma$. However, our primary motivation in this work was to assess the application of the axisymmetric similarity solution to a finite domain. Furthermore, the paper of Witkowski et al. refers to experiments (albeit as a private communication) that find an axisymmetric instability as the first transition. Finally, as with any complex dynamical system, a full understanding only comes from consideration of all states, both stable and unstable. We may also note that it is easy to generate a two-disk analogue of the non-axisymmetric, exact, von Kármán solution provided by Hewitt and Duck [18], and it would be of interest to relate this state to finite-domain computations.

Throughout this work we have not discussed the concepts of linear spatial stability, our primary concern being to describe sensible choices of edge conditions that are nonlinear perturbations of the similarity 
form. However, if one is prepared to consider linearised perturbations away from the von Kármán type of flow, then it is possible to consider a steady spatial analysis by seeking perturbations in the form

$u(r, z)=r u_{\mathrm{ss}}(z)+\epsilon r^{1+\lambda} \tilde{u}(z)$,

with similar forms for the other dependent variables and $|\epsilon| \ll 1$. (This approach is only formally valid when $\Gamma \gg 1$.) In this way one can formulate a linear eigenvalue problem for the (complex) quantity $\lambda$, and in general the set $\left\{\lambda_{i}\right\}$ will involve infinitely many values both to the left and to the right of the imaginary axis. Such an analysis has been considered in a number of papers, in particular for Jeffery-Hamel flow (see for example [19]). In the case of rotating-disk flows, one can go further, allowing for spatio-temporal development. It is a trivial observation that any pitchfork bifurcation within the von Kármán similarity form, must also lead to one of the eigenvalues $\lambda_{j}$ crossing through the origin of the complex plane; one can relate the spatial and temporal instabilities for modes of the form $\exp [\mathrm{i} \alpha(x-c t)]$ where $\lambda=\mathrm{i} \alpha$ and $x=\log (r)$. Correlation of our results for nonlinear perturbations to those of linearised and/or weakly nonlinear perturbations is an obvious area of future interest. The ultimate aim is to be able to determine the relevance of any given similarity solution to a finite-domain flow without having to compute the full solution.

Acknowledgements The authors gratefully acknowledge the support of Serco Assurance.

\section{References}

1. Batchelor GK (1951) Note on a class of solutions of the Navier-Stokes equations representing steady rotationallysymmetric flow. Q J Mech Appl Math 4:29-41

2. Zandbergen PJ, Dijkstra D (1987) Von Kármán swirling flows. Ann Rev Fluid Mech 19:465-491

3. Berman AS (1953) Laminar flow in channels with porous walls. J Appl Phys 24:1232-1235

4. Brady JF, Acrivos A (1981) Steady flow in a channel or tube with an accelerating surface velocity. An exact solution to the Navier-Stokes equations. J Fluid Mech 112:127-150

5. Zaturska MB, Banks WHH (2003) New solutions for flow in a channel with porous walls and/or non-rigid walls. Fluid Dyn Res 33:57-71

6. Hewitt RE, Duck PW, Al-Azhari M (2003) Extensions to three-dimensional flow in a porous channel. Fluid Dyn Res 33:17-39

7. Terrill RM (1982) An exact solution for flow in a porous pipe. Z Angew Math Phys 22:547-552

8. Riley N, Drazin PG (2006) The Navier-Stokes equations: A classification of flows and exact solutions (London Mathematical Society Lecture Note Series 334). Cambridge University Press. Cambridge, England

9. Brady JF, Durlofsky L (1987) On rotating disk flow. J Fluid Mech 175:363-394

10. Gauthier G, Gondret P, Moisy F, Rabaud M (2002) Instabilities in the flow between co- and counter-rotating disks. J Fluid Mech 473:1-21

11. Nore C, Tartar M, Daube O, Tuckerman LS (2004) Survey of instability thresholds of flow between exactly counterrotating disks. J Fluid Mech 511:45-65

12. Witkowski LM, Delbende I, Walker JS, Le Quéré P (2006) Axisymmetric stability of the flow between two exactly counter-rotating disks with large aspect ratio. J Fluid Mech 546:193-202

13. Gresho PM, Sani RL (1998) Incompressible flow and the finite element method. John Wiley \& Sons Ltd., Chichester, England

14. Cliffe KA (1983) Numerical calculations of two-cell and single-cell Taylor flows. J Fluid Mech 135:219-233

15. Harriott GM, Brown RA (1984) Flow in differentially rotated cylindrical drop at moderate Reynolds number. J Fluid Mech 144:403-418

16. Heil M, Hazel AL (2006) oomph-lib-An object-oriented multi-physics finite-element library. In: Schafer M, Bungartz H-J (eds) Fluid-structure interaction. Springer

17. Cliffe KA (1996) ENTWIFE (Release 6.3) Reference Manual. AEAT-0823.

18. Hewitt RE, Duck PW (2000) Non-axisymmetric rotating disk flows: nonlinear travelling-wave states. J Fluid Mech 413:287-316

19. Banks WHH, Drazin PG, Zaturska MB (1988) On perturbations to Jeffery-Hamel flow. J Fluid Mech 186:559-581 